\title{
LINE-SUM-SYMMETRIC SCALINGS OF SQUARE NONNEGATIVE MATRICES
}

\author{
B. Curtis EAVES
}

Department of Operations Research, Stanford University, Stanford, CA 94305, USA

\author{
Alan J. HOFFMAN \\ IBM Research Center, Yorktown Heights, NY 10598, USA
}

\section{Uriel G. ROTHBLUM}

Faculty of Industrial Engineering and Management, Technion-Israel Institute of Technology, Technion City, Haifa 32000, Israel

\section{Hans SCHNEIDER}

Department of Mathematics, University of Wisconsin, Madison, WI 53706, USA

Received 2 February 1984

Revised manuscript received 9 October 1984

Dedicated to G.B. Dantzig on the occasion of his seventieth birthday.

A square matrix is called line-sum-symmetric if the sum of elements in each of its rows equals the sum of elements in the corresponding column. Let $A$ be an $n \times n$ nonnegative matrix and let $X$ and $Y$ be $n \times n$ diagonal matrices having positive diagonal elements. Then the matrices $X A$, $X A X^{-1}$ and $X A Y$ are called a row-scaling, a similarity-scaling and an equivalence-scaling of $A$. The purpose of this paper is to study the different forms of line-sum-symmetric scalings of square nonnegative matrices. In particular, we characterize matrices for which such scalings exist and show uniqueness of similarity-scalings and uniqueness of row-scalings, up to a scalar multiple of the blocks corresponding to the classes of the given matrix.

Key words: Matrix Scalings.

\section{Introduction}

Let $A$ be an $n \times n$ nonnegative matrix. The matrix $A$ is called line-sum-symmetric if for $i=1, \ldots, n$, the sum of the elements in the $i$-th row of $A$ equals the sum of the elements in the $i$-th column of $A$. An $n \times n$ matrix $B$ is called a row-scaling, similarity-scaling or equivalence-scaling of $A$ if for some $n \times n$ diagonal matrices $X$ and $Y$ whose diagonal elements are positive, $B$ equals $X A, X A X^{-1}$ or $X A Y$, respectively.

This research was partially supported by National Science Foundation Grants MCS-81-21838, ECS-8310213, MCS-80-26132 and DMS-8320189. 
The purpose of this paper is to study the different forms of scalings of square nonnegative matrices, i.e., row-scalings, similarity-scalings and equivalence-scalings, which yield line-sum-symmetric matrices. We refer to such scalings as line-sumsymmetric scalings of the given matrix. In particular, it is shown (Theorems 1,2 , and 4) that a square nonnegative matrix has each form of line-sum-symmetric scaling if and only if it is completely reducible, i.e., if and only if it is the direct sum of irreducible matrices (see Section 2 for a formal definition of complete reducibility). The above result concerning similarity scalings was established by Hartfiel (1971). Hartfiel's proof is based on the minimization of a function which cannot be computed in practice. Our proof is simple and transparent, and is based on the minimization of a function which can be computed practically. We also show that if $A$ is a square nonnegative completely reducible matrix, then the blocks of the line-sum-symmetric row-scalings of $A$ corresponding to the classes of $A$ are unique up to a scalar multiple. Also, the line-sum-symmetric similarity-scaling of $A$ is unique (this result is implicit in Hartfiel (1971)). Corresponding uniqueness results are established for the diagonal matrices used to produce line-sum-symmetric row-scalings and similarity-scalings. Moreover, we characterize diagonal matrices yielding corresponding line-sum symmetric scaling (Theorems 1, 3 and 6). We conclude from the above results (Theorem 7 ) that the matrix $A$ has the different forms of line-sum-symmetric scalings if and only if there exists a line-sym-symmetric matrix having the same pattern of zero and nonzero elements as does $A$. Finally (Theorem 8 ) we use a result of Dantzig, Eaves and Rothblum (1985) concerning the decomposition of line-sum-symmetric matrices as the sum of simple matrices to obtain decompositions of completely reducible matrices as the sum of matrices which can be scaled to simple (i.e., scalar multiples of circuit) matrices by using the same diagonal matrices, respectively.

We emphasize that our results suggest computational approaches for the explicit computation of corresponding line-sum-symmetric scalings. In particular, we show that the calculation of line-sum-symmetric row-scalings corresponds to the calculation of the eigenvectors of eigenvalue zero of corresponding $M$-matrices. Also, we show that the calculation of line-sum-symmetric similarity-scaling and certain equivalence-scalings corresponds to the minimization of a fractional function over the positive orthant.

The computation of row scalings of square nonnegative matrices is used in Eaves (1984) to compute equilibria for pure trade markets with Cobb-Douglas utilities. Also, line-sum-symmetric similarity-scalings are used for the computation of social accounting matrices (see, King (1981) or Pratt and Thorbecke (1976)).

The results described in the preceding paragraphs, in particular those concerning similarity scalings, resemble known results concerning equivalence-scalings to doubly stochastic matrices. Equivalence-scalings to a doubly stochastic matrix have been studied extensively in the last twenty years. Initial work in this direction can be found in Sinkhorn $(1962,1964)$ where it was shown that every square strictly positive matrix has a unique doubly-stochastic equivalence-scaling. An alternative 
proof of this result was given by Menon (1967). Sinkhorn (1964) also demonstrated that arbitrary square nonnegative matrices need not have doubly-stochastic equivalence-scalings. Explicit characterizations of square nonnegative matrices for which such scalings do exist were obtained by Brualdi, Parter and Schneider (1966), and, independently, by Sinkhorn and Knopp (1967). Specifically, they showed that a square nonnegative matrix has a doubly stochastic equivalence-scaling if and only if it is the direct sum of fully indecomposable matrices i.e., matrices $B$ for which there exist permutation matrices $P$ and $Q$ such that $P B Q$ has a positive main diagonal and is irreducible (see Brualdi, Parter and Schneider (1966, Lemma 2.3)). Another proof of this result appears in Marshall and Olkin (1968). We note that the proof of our Theorem 2 (and Lemmas 2 and 3) in Section 4 is related to their arguments. The existence of equivalence-scalings having general predetermined row and column sums which are not all equal to 1 has also been studied, see Berman and Plemmons (1979, Chapter 4) for information and references.

Necessary and sufficient conditions that a matrix is a similarity-scaling or an equivalence-scaling of another matrix were obtained by Saunders and Schneider (1978, Theorem 2.1, Corollary 2.4, Theorem 3.1 and Remark 5.3) (see also, Golitschek, Rothblum and Schneider (1983, Corollaries 4.4 and 5.3)).

We note that the study of column scalings of a square nonnegative matrix $A$, i.e., matrices of the form $A X$ where $X$ is a diagonal matrix of appropriate size whose diagonal elements are positive, is not conducted explicitly. It is easily seen that such column scalings of a simply correspond to row scalings of $A^{\text {r }}$.

We introduce some notation and preliminary results in Section 2 and prove our main results concerning characterization of existence and uniqueness of line-sumsymmetric row-scalings, similarity-scalings and equivalence-scalings in Sections 3, 4 and 5, respectively. Next, in Section 6, we characterize patterns of matrices having corresponding line-sum-symmetric scalings. Finally, in Section 7, we obtain the decompositions of square nonnegative completely reducible matrices.

\section{Notation conventions and preliminaries}

Let $n$ be a given positive integer. We put $N \equiv\{1, \ldots, n\}$. For $J \subseteq N$ we denote by $|J|$ the number of elements of $J$. Let $A$ be an $n \times n$ matrix and let $J, K \subseteq N$. We denote by $A_{J K}$ the corresponding $|J| \times|K|$ submatrix of rows indexed by elements of $J$ and columns indexed by elements of $K$. We use the notation $A_{J}$ for $A_{J J}$.

A matrix $A$ is called nonnegative, written $A \geqslant 0$, if all of its entries are nonnegative. A matrix $A$ is called positive, written $A \gg 0$, if all of its elements are positive. Similar definitions apply to vectors.

The identity matrix in $\mathbb{R}^{n \times n}$ will be denoted by $I$. Also, the vector $(1, \ldots, 1)^{\Upsilon} \in \mathbb{R}^{n}$ will be denoted by $e$.

The spectral radius of a square matrix $A$, denoted $r(A)$, is defined to be the largest modulus of an eigenvalue of $A$. A matrix $A \in \mathbb{R}^{n \times n}$ is called an $M$-matrix if $A=r I-P$ for some nonnegative matrix $P$ and scalar $r$ where $r(P) \leqslant r$. 
A matrix $X \in \mathbb{R}^{n \times n}$ is called diagonal if $X_{i j}=0$ for all $i, j \in N$ where $i \neq j$. The set of all diagonal matrices in $\mathbb{R}^{n \times n}$ whose diagonal elements are positive will be denoted by $\mathscr{D}_{n}$. For a positive vector $x \in \mathbb{R}^{n}$, let $D(x)$ be the diagonal matrix in $\mathscr{D}_{n}$ having $D(x)_{i i}=x_{i}$ for each $i \in N$. In particular, $X=D(x)$ for $X \in \mathscr{D}_{n}$ if and only if $x=X e$. to A square matrix $P$ is called a pattern if all the elements of $P$ are either zero or one. For a given $n \times n$ matrix $A$ we define the pattern of $A$, written $P(A)$, to be the $n \times n$ pattern having $P(A)_{i j}=1$ if $A_{i j} \neq 0$ and $P(A)_{i j}=0$ if $A_{i j}=0$. A property of square nonnegative matrices is called a pattern property if it depends only on the pattern of the matrices, i.e., the property holds for a given matrix if and only if it holds for all matrices having the same pattern.

Let $A$ be an $n \times n$ nonnegative matrix. Motivated by the theory of Markov chains, we sometimes refer to the indices $1, \ldots, n$ as states. We say that state $i$ has access to state $j$, or that state $j$ has access from state $i$, if either $i=j$ or if there exist distinct integers $i_{0}=i, \ldots, i_{q}=j$, such that for $t=0, \ldots, q-1, A_{i_{i} i_{i+1}} \neq 0$. Two states $i$ and $j$, each having access to the other, are said to communicate. It is well known that the communication relation is an equivalence relation. Hence, we may partition the set $N$ into equivalence classes. The states in an equivalence class are those which communicate with each other. In the sequel a class will always mean a nonempty equivalence class of communicating states. Evidently, the accessibility relation and class structure depend on the matrix $A$. Whenever we use these concepts, the identity of the corresponding matrix $A$ will be clear from the context. Of course, the accessibility relation and class structure are pattern properties.

A square nonnegative matrix is called irreducible if the communication relation induces only one class. In particular, every $1 \times 1$ matrix (including the $1 \times 1$ zero matrix) is irreducible. We note that if $A$ is an $n \times n$ irreducible matrix where $n>1$, then no row of $A$ vanishes. Also, for every class $J$ of a square nonnegative matrix $A, A_{J}$ is irreducible. An $n \times n$ nonnegative matrix is irreducible if and only if there exists no set $\emptyset \neq K \subset N$ with $A_{K, N \backslash K}=0$. Of course, reducibility is a pattern property.

An $n \times n$ nonnegative matrix $A$ is called completely reducible if $i \in N$ has access to $j \in N$ if and only if $j$ has access to $i$. In particular, every irreducible matrix is completely reducible. An $n \times n$ nonnegative matrix is completely reducible if and only if for every $\emptyset \neq K \subset N, A_{N \backslash K, K}=0$ whenever $A_{K, N \backslash K}=0$. It follows that such a (completely reducible) matrix is the direct sum of unique irreducible matrices, i.e., there is a partition $J(1), \ldots, J(s)$ of $N$, unique except of the order of the subscripts, such that $A_{J(q)}$ is irreducible for $q=1, \ldots, s$ and $A_{J(p) J(q)}=0$ for $p$, $q=1, \ldots, s$ with $p \neq q$. Of course, complete reducibility is a pattern property.

Let $A$ be an $n \times n$ nonnegative matrix. We say that $A$ is line-sum-symmetric if

$$
\sum_{j=1}^{n} A_{i j}=\sum_{j=1}^{n} A_{j i}, \quad i=1, \ldots, n \text {. }
$$

We note that (2.1) can be written in matrix notation by

$$
A e=A^{\mathrm{T}} e .
$$


The following lemma shows that every square nonnegative line-sum-symmetric is completely reducible.

Lemma 1. Let $A$ be an $n \times n$ nonnegative line-sum-symmetric matrix. Then $A$ is completely reducible.

Proof. Assume that $\emptyset \neq K \subset N$ and $A_{K, N \backslash K}=0$. We conclude from the line-sumsymmetry of $A$ that

$$
\sum_{i \in K} \sum_{j \in K} A_{i j}=\sum_{i \in K} \sum_{j \in N} A_{i j}=\sum_{i \in K} \sum_{j \in N} A_{j i}=\sum_{i \in K} \sum_{j \in K} A_{j i}+\sum_{i \in K} \sum_{j \in N \backslash K} A_{j i}
$$

implying that $\sum_{i \in K} \sum_{j \in N \backslash K} A_{j i}=0$. As $A \geqslant 0$, we conclude that $A_{N \backslash K, K}=0$. This proves that $A$ is completely reducible.

An $n \times n$ matrix 1 is called a circuit matrix if there exist $q$ distinct integers in $\{1, \ldots, n\}$, say $i_{1}, \ldots, i_{q}$, such that

$$
A_{i j}= \begin{cases}1 & \text { if }(i, j) \in\left\{\left(i_{1}, i_{2}\right),\left(i_{2}, i_{3}\right), \ldots,\left(i_{q}, i_{q+1}\right)\right\}, \text { and } \\ 0 & \text { otherwise }\end{cases}
$$

where $i_{q+1} \equiv i_{1}$. Evidently, such a matrix is a pattern and is line-sum-symmetric (and therefore by Lemma 1 , completely reducible).

Let $A$ and $B$ be $n \times n$ nonnegative matrices. We say that $B$ is a row-scaling, a similarity-scaling or an equivalence-scaling of $A$ if $B=X A, B=X A X^{-1}$ or $B=X A Y$, respectively, for some matrices $X$ and $Y$ in $\mathscr{D}_{n}$. Of course, each row-scaling and each similarity-scaling of $A$ is an equivalence-scaling of $A$. We observe that the pattern of each equivalence-scaling of $A$ (and therefore of each row-scaling and each similarity-scaling of $A$ ) coincides with the pattern of $A$. Finally, the relations of being a row-scaling, of being a similarity-scaling and of being an equivalencescaling are clearly equivalence relations.

\section{Line-sum-symmetric row-scalings}

We start with our main result concerning line-sum-symmetric row-scalings.

Theorem 1. Let $A$ be an $n \times n$ nonnegative matrix. Then:

(a) $A$ has a line-sum-symmetric row-scaling if and only if $A$ is completely reducible,

(b) if $Y$ and $Z$ are in $\mathscr{D}_{n}$ where both $Y A$ and $Z A$ are line-sum-symmetric, then for every class $J$ of $A$ there exists a positive scalar $\gamma_{J}$ such that $Y_{J}=\gamma_{J} Z_{J}$, and 
(c) if $A$ satisfies the equivalent conditions of part (a) and $B$ and $C$ are two line-sum-symmetric row-scalings of $A$, then for every class $J$ of $A$ there exists a positive scalar $\gamma_{J}$ such that $B_{J}=\gamma_{J} C_{J}$.

Proof. First assume that $A$ has a line-sum-symmetric scaling $B$. Then $A$ and $B$ have the same pattern. Also, by Lemma $1, B$ is completely reducible. Thus, as complete reducibility is a pattern property, $A$ is also completely reducible.

We next show that if $A$ is completely reducible then $A$ has a line-sum-symmetric row-scaling. First consider the case where $A$ is irreducible. Let

$$
r \equiv \max _{i \in N}\left(\sum_{j \in N \backslash\{i\}} A_{i j}\right)
$$

and let $P$ be the $n \times n$ matrix having

$$
P_{i j}= \begin{cases}A_{i j} & \text { if } i, j \in N \text { where } i \neq j, \text { and } \\ r-\sum_{i \in N \backslash\{i\}} A_{i l} & \text { if } i, j \in N \text { where } i=j .\end{cases}
$$

Evidently, $P$ is nonnegative and irreducible (as $A$ has these properties). Also, $P e=r e$. It follows from Berman and Plemmons (1979, Corollary 2.1.12) that the spectral radius of $P$ is $r$ and therefore the Perron-Frobenius Theorem (e.g., Berman and Plemmons (1979, Theorem 2.1.4)) implies that for some positive vector $x \in \mathbb{R}^{n}$, $x^{\mathrm{T}} P=r x^{\mathrm{T}}$. In particular, for $j \in N$,

$$
\sum_{k \in N \backslash j\}} x_{k} A_{k j}+x_{j}\left(r-\sum_{t \in N \backslash\{j\}} A_{j t}\right)=r x_{j},
$$

implying that

$$
\sum_{k \in N} x_{k} A_{k j}=\sum_{i \in N} x_{j} A_{j t}
$$

In particular, if $X=D(x)$, then $X A$ is line-sum-symmetric. The case where $A$ is an (arbitrary) completely reducible matrix follows directly from the (established) result for irreducible matrices and the fact that each completely reducible matrix is the direct sum of irreducible matrices.

We next establish parts (b) and (c) in the case where $A$ is irreducible. Assume that $Y$ and $Z$ are in $\mathscr{D}_{n}$ where both $Y A$ and $Z A$ are line-sum-symmetric. Let $y \equiv Y e$ and $z=Z e$. It follows from the line-sum-symmetry of $A$ that $y$ and $z$ satisfy (3.4) and therefore (3.3) (with $y$ and $z$ replacing $x$, respectively). Thus, $y^{\mathrm{T}} P=r y^{\mathrm{T}}$ and $z^{\mathrm{T}} P=r z^{\mathrm{T}}$, where $P$ is defined by (3.2). As $P$ is irreducible we have from the Perron-Frobenius Theorem (e.g., Berman and Plemmons (1979, Theorem 2.1.4)) that for some positive scalar $\gamma, y=\gamma z$, i.e., $Y=\gamma Z$. In particular, $Y A=\gamma(Z A)$. This establishes parts (b) and (c) in the case where $A$ is irreducible. The proof of these results for the case where $A$ is completely reducible follows from the fact that a completely reducible matrix is the direct sum of irreducible matrices. 
Our proof of Theorem 1 shows that the problem of computing a line-sumsymmetric row-scaling of a square nonnegative irreducible matrix $A$ corresponds to the computation of a positive eigenvector corresponding to eigenvalue zero of (the $M$-matrix) $r I-P$, where $r$ and $P$ are defined by (3.1) and (3.2), respectively. Of course, for a completely reducible matrix $A$, one has to compute one such eigenvector for each class of $A$.

In general we do not have an explicit expression for the unique, up to scalar multiple, line-sum-symmetric row-scaling of a given $n \times n$, nonnegative, completely reducible matrix $A$. However, in the special case where the pattern of $A$ is a circuit matrix, say $P, P$ is a line-sum-symmetric row-scaling of $A$. Moreover, if $\{(i, j) \in N \times$ $\left.N: P_{i j}=1\right\}=\left\{\left(i_{1}, i_{2}\right), \ldots,\left(i_{q}, i_{q+1}\right)\right\}$ where $i_{1}, \ldots, i_{q}$ are distinct integers and where $i_{q+1}=i_{1}$, then, trivially, $X A=P$ for any matrix $X \in \mathscr{D}_{n}$ having

$$
X_{i, i_{i}}=\left(A_{i_{i} i_{t+1}}\right)^{-1}, \quad t=1, \ldots, q .
$$

Corollary 1. Let $A$ be an $n \times n$ nonnegative matrix. Then there exists a nonzero $n \times n$ diagonal matrix $X$ whose diagonal elements are nonnegative, such that $X A$ is line-sumsymmetric. Moreover, whenever $X$ is such a matrix, $K \equiv\left\{i \in N: X_{i i} \neq 0\right\}$ is a union of classes of $A$ and $A_{k}$ is completely reducible.

Parts (b) and (c) of Theorem 1 can be used to obtain corresponding uniqueness results in Corollary 1.

\section{Line-sum-symmetric similarity-scalings}

Before developing our main result concerning line-sum-symmetric similarityscalings, we state and prove two auxiliary lemmas.

Lemma 2. Let $A$ be an $n \times n$ irreducible nonnegative matrix and let

$$
a \equiv \min \left\{A_{i j}: i, j \in N, A_{i j} \neq 0\right\} .
$$

Also, let $f$ be the real valued function defined on $\Omega=\left\{x \in \mathbb{R}^{n}: x \gg 0\right\}$ by

$$
f(x)=\sum_{i=1}^{n} \sum_{j=1}^{n} x_{i} A_{i j} x_{j}^{-1}
$$

Then:

(a) $f$ is homogenous of degree zero, i.e.,

$$
f(\gamma x)=f(x) \text { for every } x \in \Omega \text { and } \gamma>0,
$$

(b) for every $x \in \Omega$ with $\sum_{i=1}^{n} x_{i}=1$,

$$
f(x) \geqslant a n^{-1 / n}\left(\min _{i \in N} x_{i}\right)^{-1 / n},
$$

and

(c) the function $f$ attains a minimum over $\Omega$. 
Proof. Part (a) is immediate from the definition of $f$. Also, part (b) is trivial in the case where $n=1$. We next establish part (b) under the assumption that $n>1$. Let $x \in \Omega$ satisfy $\sum_{i=1}^{n} x_{i}=1$. Then there exist integers $p$ and $k$ in $N$ where $p \neq k, x_{p} \geqslant n^{-1}$ and $x_{k}=\min _{i \in N} x_{i}$. In particular, $x_{p} \geqslant x_{k}$. Now, as $A$ is irreducible, $p$ has access to $k$. Thus, there exist distinct integers $i_{0}=p, i_{1}, \ldots, i_{q}=k$ such that for $t=0, \ldots, q-1$, $A_{i_{r} i_{t+1}} \neq 0$. Using the fact that the arithmetic mean is larger or equal than the geometric mean we conclude that

$$
\begin{aligned}
f(x) & \geqslant q^{-1} f(x) \geqslant q^{-1}\left(\sum_{t=0}^{q-1} x_{i_{t}} A_{i_{i} i_{t+1}} x_{i_{t+1}}^{-1}\right) \geqslant\left(\prod_{t=0}^{q-1} x_{i_{t}} A_{i_{t} i_{t+1}} x_{i_{t+1}}^{-1}\right)^{1 / q} \\
& \geqslant\left(\prod_{t=0}^{q-1} x_{i_{t}} a x_{i_{t+1}}^{-1}\right)^{1 / q}=a\left(x_{p} x_{k}^{-1}\right)^{1 / q} \geqslant a\left(x_{p} x_{k}^{-1}\right)^{1 / n} \geqslant a n^{-1 / n} x_{k}^{-1 / n} .
\end{aligned}
$$

establishing part (b).

We finally establish part (c). Let $\delta \equiv n^{-1}\left[a f(e)^{-1}\right]^{n}$, let

$$
\Omega^{*} \equiv\left\{x \in \Omega: \sum_{i=1}^{n} x_{i}=1\right\}
$$

and let

$$
\Omega_{\delta}^{*} \equiv\left\{x \in \Omega^{*}: \min _{i \in N} x_{i} \geqslant \delta\right\}
$$

For $x \in \Omega^{*} \backslash \Omega_{\delta}^{*}, \min _{i \in N} x_{i}<\delta$ and parts (b) and (a) imply that

$$
f(x) \geqslant a n^{-1 / n}\left(\min _{i \in N} x_{i}\right)^{-1 / n}>a n^{-1 / n} \delta^{-1 / n}=f(e)=f\left(n^{-1} e\right) .
$$

As $A$ is irreducible no row of $A$ vanishes. It follows that $f(e) \geqslant n a$, implying that $\delta \leqslant n^{-1}$. In particular, $n^{-1} e \in \Omega_{\delta}^{*}$. It follows that

$$
\inf _{x \in \Omega \text { 促 }} f(x)=\inf _{x \in \Omega^{*}} f(x)=\inf _{x \in \Omega} f(x)
$$

since the second equality is clear. As $\Omega_{\delta}^{*}$ is nonempty and compact and $f$ is continuous (on $\Omega$ ), we conclude that $f$ attains a minimum over $\Omega_{\delta}^{*}$, and therefore, by (4.5), also over $\Omega$.

We note that under the assumptions of Lemma 2, the function $f$ tends to infinity uniformly as $x$ approaches the boundary of $\Omega$.

Our next lemma characterizes square nonnegative matrices $A$ for which the function $f$ defined by (4.2) attains a minimum. Specifically, it is shown that these matrices are precisely the completely reducible matrices.

Lemma 3. Let $A$ be an $n \times n$ nonnegative matrix and let $f$ be the real valued function defined by (4.2) on the set $\Omega=\left\{x \in \mathbb{R}^{r}: x \gg 0\right\}$. Then the following are equivalent:

(a) $f$ attains a minimum over $\Omega$, and

(b) $A$ is completely reducible. 
Moreover, if $f$ attains a minimum over $\Omega$ at $\tilde{x} \in \Omega$, then, for each $i \in N$,

$$
\sum_{j=1}^{n} \tilde{x}_{i} A_{i j} \tilde{x}_{j}^{-1}=\sum_{j=1}^{n} \tilde{x}_{j} A_{j i} \tilde{x}_{i}^{-1}
$$

Proof. If $A$ is irreducible, part (c) of Lemma 2 implies that $f$ attains a minimum over $\Omega$. The extension of this conclusion to the case where $A$ is completely reducible is straight forward. Next assume that $f$ attains a minimum over $\Omega$ and let this minimum be attained at $\tilde{x}$. Suppose $A$ is not completely reducible. Then $N$ may be partitioned into two set $I$ and $J$ such that $A_{j i}=0$ for each $i \in J$ and $j \in J$ and where there exist $k \in I$ and $h \in J$ such that $A_{k h}>0$. Let $x \in \Omega$ be defined by $x_{i}=\varepsilon \tilde{x}_{i}$ if $i \in I$ and $x_{j}=\tilde{x}_{j}$ if $j \in J$ where $0<\varepsilon<1$. Then

$$
f(x)=f(\tilde{x})+\sum_{\substack{i \in I \\ j \in J}}\left(x_{i} A_{i j} x_{j}^{-1}-\tilde{x}_{i} A_{i j} \tilde{x}_{j}^{-1}\right)=f(\tilde{x})+(\varepsilon-1)\left(\sum_{i=I} \tilde{x}_{i} A_{i j} \tilde{x}_{j}^{-1}\right)<f(\tilde{x}),
$$

contradicting the minimality of $\tilde{x}$. This contradiction proves that $A$ is, indeed, completely reducible.

Finally, assume that $f$ attains a minimum over $\Omega$ at $\tilde{x}$. Since $f$ is differentiable over the open set $\Omega$, we conclude that the gradient of $f$ at $x$ vanishes, i.e., for $k=1, \ldots, n$,

$$
0=\frac{\partial f}{\partial x_{k}}(\tilde{x})=\sum_{\substack{j=1 \\ j \neq 1}}^{n} A_{k j} \tilde{x}_{j}^{-1}=\sum_{\substack{i=1 \\ i \neq k}}^{n} \tilde{x}_{i} A_{i k} \tilde{x}_{k}^{-2},
$$

implying that

$$
\sum_{j=1}^{n} \tilde{x}_{k} A_{k j} \tilde{x}_{j}^{-1}=\sum_{i=1}^{n} \tilde{x}_{i} A_{i k} \tilde{x}_{k}^{-1}
$$

We are now ready for the two main results concerning line-sum-symmetric similarity-scalings. The first result (Theorem 2) characterizes square nonnegative matrices having line-sum symmetric similarity-scalings. The second (Theorem 3 ) characterizes the diagonal matrices which yield such scalings (for a given square nonnegative matrix). The first result appears in Hartfiel (1971). Our proof is simple and our approach suggests a practical method for computing the corresponding line-sum-symmetric similarity-scalings.

Theorem 2. Let $A$ be an $n \times n$ nonnegative matrix. Then:

(a) $A$ has a line-sum-symmetric similarity-scaling if and only if $A$ is completely reducible.

(b) if $Y$ and $Z$ are in $\mathscr{D}_{n}$ where both $Y A Y^{-1}$ and $Z A Z^{-1}$ are line-sum-symmetric, then for every class $J$ of $A$ there exists a positive scalar $\gamma_{J}$ such that $Y_{J}=\gamma_{J} Z_{J}$ and

(c) if $A$ satisfies the equivalent conditions of part (a), then A has a unique line-sumsymmetric similarity-scaling. 
Proof. The fact that if $A$ has a line-sum-symmetric similarity-scaling then $A$ is completely reducible follows from the arguments used to prove the corresponding result concerning row-scalins in Theorem 1. We next show that if $A$ is completely reducible then $A$ has a line-sum-symmetric similarity-scaling. Suppose $A$ is completely reducible. Lemma 3 shows the existence of positive elements $x_{1}, \ldots, x_{n}$ for which (4.6) holds. In particular, for $X=D(\tilde{x})$, we have that $X A X^{-1}$ is line-sumsymmetric.

We next establish parts (b) and (c) in the case where $A$ is irreducible. Assume that $Y$ and $Z$ are two matrices in $\mathscr{D}_{n}$ where $B \equiv Y A Y^{-1}$ and $C \equiv Z A Z^{-1}$ are line-sum-symmetric. Evidently, as $A$ is irreducible so are $B$ and $C$. Let $W=Y Z^{-1}$. Then $W \in \mathscr{D}_{n}$ and $B=W C W^{-1}$. Let $\gamma \equiv \max \left\{W_{i i}: i=1, \ldots, n\right\}$ and $J \equiv$ $\left\{i=1, \ldots, n: W_{i i}=\gamma\right\}$. Evidently, $J \neq \emptyset$. Let $i \in J$. By the line-sum-symmetry of $B$,

$$
\sum_{j \in N} W_{i i} C_{i j} W_{j j}^{-1}=\sum_{j \in N} B_{i j}=\sum_{j \in N} B_{j i}=\sum_{j \in N} W_{j j} C_{j i} W_{i i}^{1}
$$

implying that

$$
\begin{aligned}
\gamma^{2} & =W_{i i}^{2}=\left(\sum_{j \in N} W_{j j} C_{j i}\right) /\left(\sum_{j \in N} C_{i j} W_{j j}^{-1}\right) \\
& \leqslant\left(\sum_{j \in N} \gamma C_{j i}\right) /\left(\sum_{j \in N} C_{i j} \gamma^{-1}\right)=\gamma^{2}\left(\sum_{j \in N} C_{j i}\right) /\left(\sum_{j \in N} C_{i j}\right)=\gamma^{2},
\end{aligned}
$$

where the last equality follows from the line-sum-symmetry of $C$. It follows that if $j \in N \backslash J$, i.e., $W_{j j}<\gamma$, then $C_{i j}=C_{j i}=0$. We next show that $J=N$. If this is not the case and $J \subset N$, then $\emptyset \neq J \subset N$ with $C_{J, N \backslash J}=0$ and $C_{N / J, J}=0$, contradicting the irreducibility of $C$. It follows that $Y Z^{-1}=W=\gamma I$, i.e., $Y=\gamma Z$. In particular, $B=$ $Y A Y^{-1}=Z A Z^{-1}=C$. Finally, the proof of parts (b) and (c) for reducible matrices follows directly from the (established) corresponding results for irreducible matrices.

We next combine part (a) of Theorem 2 with Lemma 3 to characterize existence of line-sum-symmetric similarity-scalings of square nonnegative matrices in terms of the minimization problem considered in Lemmas 2 and 3.

Corollary 2. Let $A$ be an $n \times n$ nonnegative matrix and let $f$ be the real valued function defined on $\Omega\left\{x \in \mathbb{R}^{n}: x \gg 0\right\}$ by (4.2). Then the following are equivalent:

(a) $A$ has a line-sum-symmetric similarity scaling,

(b) $A$ is completely reducible, and

(c) f attains a minimum over $\Omega$.

Proof. The equivalence of (a) and (b) follows from part (a) of Theorem 2 and the equivalence of (b) and (c) follows from Lemma 3. 
We next obtain a characterization of the set of diagonal matrices yielding line-sumsymmetric similarity-scalings of square nonnegative matrices in terms of the minimization problem considered in Lemmas 2 and 3 . We then use this corollary to obtain a characterization of line-sum-symmetric matrices.

Theorem 3. Let $A$ be an $n \times n$ negative matrix and let $f$ be the real valued function defined on $\Omega=\left\{x \in \mathbb{R}^{n}: x \gg 0\right\}$ by (4.2). Consider the following properties of a vector $\tilde{x} \in \Omega$ :

(a) $\tilde{x}$ minimizes the function $f$ over $\Omega$, and

(b) the matrix $D(\tilde{x}) A D(\tilde{x})^{-1}$ is line-sum symmetric.

The $\tilde{x}$ satisfies (a) if and only if $\tilde{x}$ satisfies (b). Moreover, if there exists a vector $\tilde{x}$ satisfying (a), or equivalently (b), then for each class $J$ of $A$ there exists a vector $x^{J} \in \mathbb{R}^{n}$ with $\left(x^{J}\right)_{J}>0$ and $\left(x^{J}\right)_{i}=0$ for $i \in N \backslash J$ such that the set of vectors $\tilde{x}$ satisfying (a), or equivalently (b), has the form $\left\{\sum_{J} \gamma_{J} x^{J}: \gamma_{J}>0\right.$ for each class $J$ of $\left.A\right\}$.

Proof. The arguments of the proof of Lemma 3 show that if $\tilde{x} \in \Omega$ satisfies (a) then $\tilde{x}$ must satisfy (b). Next assume that $\tilde{x}$ satisfies (b). Then, by Corollary $2, A$ is completely reducible and there exists a vector $x^{*} \in \Omega$ which minimizes $f$ over $\Omega$. By the established implication (a) $\Rightarrow(\mathrm{b}), D\left(x^{*}\right) A D\left(x^{*}\right)^{-1}$ is line-sum-symmetric. It now follows from part (b) of Theorem 2 that for every class $J$ of $A$ there exists a positive number $\gamma_{J}$ such that $D(\tilde{x})_{J}=\gamma_{J} D\left(x^{*}\right)_{J}$. Thus, $\tilde{x}_{J}=\gamma_{J} x_{J}^{*}$ for each class $J$ of $A$. It now follows immediately from the complete reducibility of $A$ that $f(x)=f\left(x^{*}\right)$, implying that $\tilde{x}$, like $x^{*}$, minimizes $f$ over $\Omega$, i.e., $\tilde{x}$ satisfies (a).

Next assume that $x^{*}$ satisfies (a), or equivalently (b). In particular, it follows from Corollary 2 that $A$ must be completely reducible. Now for each class $J$ of $A$, let $x^{J}$ be the vector in $\mathbb{R}^{n}$ having $\left(x^{J}\right)_{J}=\left(x^{*}\right)_{J}$ and $\left(x^{J}\right)_{i}=0$ for $i \in N \backslash J$. By the complete reducibility of $A$ we have that if $x \in\left\{\sum_{J} \gamma_{J} x^{J}: \gamma_{J}>0\right.$ for each class $J$ of $A\} \subseteq \Omega$ then $f(x)=f\left(x^{*}\right)$, assuring that such $x$ minimizes $f$ over $\Omega$. Then our earlier arguments imply that $D(x) A D(x)^{-1}$ is line-sum-symmetric. As $D\left(x^{*}\right) A D\left(x^{*}\right)^{-1}$ is also line-sum-symmetric we have from part (b) of Theorem 2 that for each class $J$ of $A$ there exists a positive number $\gamma_{J}$ such that $D(x)_{J}=D\left(x^{*}\right)_{J}$, or equivalently, $x_{J}=\gamma_{J} x_{J}^{*}$. It immediately follows that $x=\sum_{J} \gamma_{J} x^{J}$ where $J$ ranges over the classes of $A$. This completes our proof of the desired representation of the set of vectors in $\Omega$ satisfying (a) or equivalently (b).

Theorem 3 shows that the problem of searching for a line-sum-symmetric similarity-scaling of a given square, nonnegative matrix can be transformed to the problem of minimizing a (nonlinear) function over the positive orthant. Corollary 2 shows that these problems have a solution if and only if the underlying matrix is completely reducible. In this case, Lemma 2 shows that the objective function of the corresponding minimization problem is uniformly unbounded near the boundary of the nonnegative orthant. In particular, since setting $x_{j}=e^{y_{j}}$ transforms $f(x)$ into a convex function $g(y)$, methods of nonlinear programming can be used for computing line-sum-symmetric similarity-scalings whenever such scalings exist. 
We next use our results to characterize line-sum-symmetric matrices.

Corollary 3. Let $A$ be an $n \times n$ nonnegative matrix. Then $A$ is line-sum-symmetric if and only

$$
\sum_{i=1} \sum_{j=1} x_{i} A_{i j} x_{j}^{-1} \geqslant \sum_{i=1}^{n} \sum_{j=1}^{n} A_{i j}
$$

for all $x \gg 0$. Moreover, if $A$ is irreducible and line-sum-symmetric, equality in (4.8) holds if and only if all the coordinates of $x$ coincide.

Proof. Evidently, (4.8) holds for all $x \gg 0$ if and only if the function $f$ defined by (4.2) on $\Omega=\left\{x \in \mathbb{R}^{n}: x \gg 0\right\}$ attains a minimum at $e$. By Theorem 3, this occurs if and only if $A=I A I^{-1}=D(e) A D(e)^{-1}$ is line-sum symmetric. Next assume that $A$ is irreducible and line-sum-symmetric. Then $A$ has a single class and Theorem 3 implies that the set of vectors minimizing the function $f$ over $\Omega$ has the form $\left\{\gamma x^{*}: \gamma>0\right\}$ for some $x^{*} \in \Omega$. Our earlier arguments show that $e$ is in this class. It immediately follows that $\left\{\gamma x^{*}: \gamma>0\right\}=\{\delta e: \delta>0\}$, implying that the set of vectors minimizing $f$ over $\Omega$ is the set of vectors all of whose coordinates coincide. It follows that equality holds in (4.8) if and only if all the coordinates of $x$ coincide.

In general we do not know of an explicit expression for the unique line-sumsymmetric similarity-scaling of a given $n \times n$ nonnegative completely reducible matrix $A$. However, in the special case where the pattern of $A$ is a circuit matrix, say $P$, with $\left\{(i, j) \in N \times N: P_{i j}=1\right\}=\left\{\left(i_{1}, i_{2}\right), \ldots,\left(i_{q}, i_{q+1}\right)\right\}$, where $i_{1}, \ldots, i_{q}$ are distinct integers and where $i_{q+1} \equiv i_{1}$, the unique line-sum-symmetric similarity scaling of $A$ is the matrix $\alpha P$ where $\alpha=\left(\prod_{t=1}^{q} A_{i_{i} i_{t+1}}\right)^{1 / q}$. Moreover, $X A X^{-1}=\alpha P$ for any matrix $X \in \mathscr{D}_{n}$

$$
X_{i, i_{i}}=\alpha^{-t+1}\left[\prod_{u=1}^{t-1} A_{i_{u} i_{u+1}}\right], \quad t=1, \ldots, q .
$$

Verification of this assertion can be done by direct substitution (cf., Engel and Schneider (1973, Theorem 4.1, and 1975, Theorem 7.2) where a more general result is given).

\section{Line-sum-symmetric equivalence-scalings}

We next consider line-sum-symmetric equivalence-scalings.

Theorem 4. Let $A$ be an $n \times n$ nonnegative matrix. Then

(a) $A$ has a line-sum-symmetric equivalence-scaling if and only if $A$ is completely reducible, and 
(b) if $X, Y$ and $Z$ are matrices in $\mathscr{D}_{n}$ where both $Y A X$ and $Z A X$ are line-sumsymmetric, then for every class $J$ of $A$ there exists a positive scalar $\gamma_{J}$ such that $Y_{J}=\gamma_{J} Z_{J}$, and

(c) if A satisfies the equivalent conditions of part (a) and $X$ is a matrix in $\mathscr{D}_{n}$, then there exists a matrix $Y \in \mathscr{D}_{n}$ for which $Y A X$ is a line-sum-symmetric; moreover, if for $Y, Z \in \mathscr{D}_{n}$ both $B \equiv Y A X$ and $C \equiv Z A X$ are line-sum-symmetric, then for every class $J$ of $A$ there exists a positive scalar $\Gamma_{J}$ such that $B_{J}=\gamma_{J} C_{J}$.

Proof. The fact that if $A$ has a line-sum-symmetric equivalence-scaling then $A$ is completely reducible follows from the arguments used to prove the corresponding result concerning row-scalings in Theorem 1 . Alternatively, assume that $A$ is completely reducible. Let $X$ be any matrix in $\mathscr{D}_{n}$. Then $A X$ is completely-reducible and therefore, by Theorem 1, there exists a matrix $Y$ in $\mathscr{D}_{n}$ for which $X A Y$ is line-sumsymmetric. This completes the proof of (a). The remaining parts (b) and (c) follows from the above arguments and corresponding (uniqueness) results in Theorem 1 .

The following example demonstrates that a square nonnegative matrix can have many line-sum-symmetric equivalence-scalings. Let

$$
A=\left(\begin{array}{ll}
1 & 1 \\
1 & 1
\end{array}\right) \text { and } X^{\beta}=\left(\begin{array}{ll}
1 & 0 \\
0 & \beta
\end{array}\right), \quad \beta \in \mathbb{R}
$$

Then,

$$
X^{\beta} A X^{\beta}=\left(\begin{array}{cc}
1 & \beta \\
\beta & \beta^{2}
\end{array}\right)
$$

is line-sum-symmetric for every $\beta \in \mathbb{R}$.

Corollary 4. Let $A$ be an $n \times n$ nonnegative matrix. Then for every $n \times n$ diagonal matrix $Y$ whose diagonal elements are nonnegative, there exists a nonzero, $n \times n$ diagonal matrix $X$ whose diagonal elements are nonnegative, such that $X A Y$ is line-sum-symmetric. Moreover, when $X$ is such a matrix, $K \equiv\left\{i \in N: X_{i i}=0\right\}$ is a union of classes if $A Y$ and $(A Y)_{K}$ is completely reducible.

Parts (b) and (c) of Theorem 4 can be used to obtain corresponding uniqueness results in Theorem 3 and 4 Corollary 4.

We next study line-sum-symmetric equivalence-scalings for which the corresponding diagonal matrices satisfy certain constraints. We first establish a simple lemma that relates such (constrained) equivalence-scalings to similarity-scalings of a related matrix.

Lemma 4. Let $A$ be an $n \times n$ nonnegative matrix and let $d$ be a positive vector in $\mathbb{R}^{n}$. Also, let $X, Y \in \mathscr{D}_{n}$ where $X Y=D(d)$. Then $B=X A Y$ if and only if $B=$ $X[A D(d)] X^{-1}$ and $Y=D(d) X^{-1}$. 
Proof. The result is immediate by direct substitution.

Theorem 5. Let $A$ be an $n \times n$ nonnegative matrix and let $d$ be a positive vector in $\mathbb{R}^{n}$. Then:

(a) $A$ has a line-sum-symmetric equivalence-scaling $X A Y$ where $X, Y \in \mathscr{D}_{n}$ and $X Y=D(d)$ if and only if $A$ is completely reducible,

(b) if $X, Y, X^{\prime}, Y^{\prime}$ are in $\mathscr{D}_{n}$ where $X A Y$ and $X^{\prime} A Y^{\prime}$ are line-sum-symmetric and $X Y=X^{\prime} Y^{\prime}=D(d)$, then for every class $J$ of $A$ there exists a positive scalar $\gamma_{J}$ such that $X_{J}^{\prime}=\gamma_{J} X_{J}$ and $Y_{J}^{\prime}=\left(\gamma_{J}\right)^{-1} Y_{J}$, and

(c) if $A$ satisfies the equivalent conditions of part (a), then $A$ has a unique line-sumsymmetric equivalence-scaling $X A Y$ for which $X Y=D(d)$.

Proof. The conclusions of our theorem are immediate from Lemma 4, Theorem 2 and the observation that $A$ is completely reducible if and only if so is $A D(d)$.

We next obtain a characterization of the diagonal matrices yielding line-sumsymmetric equivalence-scalings of square nonnegative matrices in terms of certain minimization problems.

Theorem 6. Let $A$ be an $n \times n$ nonnegative matrix and let $d$ be a positive vector in $\mathbb{R}^{n}$. Let $g$ be the real valued function defined on

$$
\Lambda^{d}=\left\{(x, y): x, y \in \mathbb{R}^{n}, x, y \gg 0 \text { and } x_{i} y_{i}=d_{i} \text { for } i=1, \ldots, n\right\}
$$

by

$$
g(x, y)=x^{\mathrm{T}} A y .
$$

Consider the following properties of pair of vectors $(\tilde{x}, \tilde{y}) \in \Lambda^{d}$ :

(a) $(\tilde{x}, \tilde{y})$ minimizes the function $g$ over $\Lambda^{d}$, and

(b) the matrix $D(\tilde{x}) A D(\tilde{y})$ is line-sum-symmetric.

Then $(\tilde{x}, \tilde{y})$ satisfies (a) if and only if $(\tilde{x}, \tilde{y})$ satisfies (b). Moreover, if there exists a pair of vectors $(\tilde{x}, \tilde{y})$ satisfying (a), or equivalently (b), then for each class $J$ of $A$ there exists pairs of vectors $\left(x^{J}, y^{J}\right) \in \mathbb{R}^{n} \times \mathbb{R}^{n}$ with $\left(x^{J}\right)_{i}\left(y^{J}\right)_{i}=d_{i}$ for $i \in J$ and $\left(x^{J}\right)_{i}=$ $\left.y^{J}\right)_{i}=0$ for $i \in N \backslash J$, such that the set of pairs of vector $(\tilde{x}, \tilde{y}) \in \Lambda^{d}$ satisfying (a), or equivalently (b), has the form $\left\{\sum_{J}\left(s_{J} x^{J}, \gamma_{J}^{-1} y^{J}\right): \gamma_{J}>0\right.$ for each class $J$ of $\left.A\right\}$.

Proof. Conditions (a) and (b) are equivalent, respectively, to the following conditions:

( $\left.\mathrm{a}^{\prime}\right) \tilde{x}$ minimizes the real valued function of defined on $\Omega \equiv\left\{x \in \mathbb{R}^{n}: x>0\right\}$ by

$$
f(x)=\sum_{i=1}^{n} \sum_{j=1}^{n} x_{i}[A D(d)]_{i j} x_{j}^{-1}
$$

and $\tilde{y}_{i}=d_{i} \tilde{x}_{i}^{-1}$ for $i=1, \ldots, n$, and

(b') the matrix $D(\tilde{x}) A D(d) D(\tilde{x})^{-1}$ is line-sum-symmetric and $\tilde{y}_{i}=d_{i} \tilde{x}_{1}^{-1}$ for $i=$ $1, \ldots, n$. 
(See Lemma 1.) It was shown in Theorem 3 that $\left(a^{\prime}\right)$ and $\left(b^{\prime}\right)$ are equivalent and a representation of the set of vectors satisfying $\left(a^{\prime}\right)$ and $\left(b^{\prime}\right)$ was given there. The conclusion of our theorem now follows easily by direct substitution.

Observe that condition (b) of Theorem 6 states that for $i=1, \ldots, n$

$$
x_{i}(A y)_{i}=\left(x^{\mathrm{T}} A\right)_{i} y_{i}
$$

or equivalently,

$$
(A y)_{\mathrm{i}} / y_{\mathrm{i}}=\left(A^{\mathrm{T}} x\right)_{\mathrm{i}} / x_{\mathrm{i}}
$$

We next use Theorem 5 to obtain a number of inequalities that are associated with certain line-sum-symmetric matrices.

Corollary 5. Let $A$ be an $n \times n$ nonnegative matrix and let $u$ and $v$ be positive vectors in $\mathbb{R}^{n}$. Then $D(u) A D(v)$ is line-sum-symmetric if and only if

$$
x^{\mathrm{T}} A y \geqslant u^{\mathrm{T}} A v
$$

for all pairs of positive vectors $(x, y) \in \mathbb{R}^{n} \times \mathbb{R}^{n}$ for which $x_{i} y_{i}=u_{i} v_{i}$ for $i=1, \ldots, n$. Further, if $D(u) A D(v)$ is line-sum-symmetric and $A$ is irreducible, the equality holds in (5.4) if and only if $x=\gamma u$ and $y=\gamma^{-1} v$ for some $\gamma>0$.

Proof. Let $d$ be the vector in $\mathbb{R}^{n}$ having $d_{i}=u_{i} v_{i}$ for $i=1, \ldots, n$. Then

$$
(u, v) \in \Lambda^{d}=\left\{(x, y): x, y \in \mathbb{R}^{n}, y, y \gg 0 \text { and } x_{i} y_{i}=d_{i} \text { for } i=1, \ldots, n\right\} .
$$

It now follows from Theorem 6 that $D(u) A D(v)$ is line-sum-symmetric if and only if $(u, v)$ satisfies $(5.4)$ for all pairs of vectors $(x, y) \in \Lambda^{d}$. Further, it follows from Theorem 6 that if the above two equivalent conditions hold and $A$ is irreducible then the set of minimizers of the corresponding minimization problem defined in Theorem 6 has the form $\left\{\left(\gamma u, \gamma^{-1} v\right): \gamma>0\right\}$ implying that equality in (5.4) holds if and only if $x=\gamma u$ and $v=\gamma^{-1} v$ for some $\gamma>0$.

Corollary 6 (Fiedler, Johnson, Markham and Neumann (1985)). Let $A$ be an $n \times n$ nonnegative matrix and let $u$ and $v$ be positive left and right eigenvectors, respectively, of $A$ corresponding to $r(A)$. Then

$$
x^{\mathrm{T}} A y \geqslant u^{\mathrm{T}} A v
$$

for all pairs of positive vectors $(x, y) \in \mathbb{R}^{n} \times \mathbb{R}^{n}$ for which $x_{i} y_{i}=u_{i} v_{i}$ for $i=1, \ldots, n$.

Proof. If $u$ and $v$ are positive left and right eigenvectors, respectively, of $A$ corresponding to $r(A)$, then for $i=1, \ldots, n$

$$
\left(u^{T} A\right)_{i} v_{i}=u_{i} r(A) v_{i}=u_{i}(A v)_{i}
$$

implying that $D(u) A D(v)$ is line-sum-symmetric (see the paragraph following 
Theorem 6). It now follows directly from Corollary 5 that (5.5) holds for all corresponding pairs of vectors $(x, y)$.

The converse of Corollary 6 is false even for irreducible matrices. For example, let

$$
A=\left(\begin{array}{ll}
1 & 1 \\
1 & 1
\end{array}\right), \quad u=\left(\begin{array}{l}
1 \\
2
\end{array}\right), \quad v=\left(\begin{array}{l}
1 \\
2
\end{array}\right)
$$

Then

$$
D(u) A D(v)=\left(\begin{array}{ll}
1 & 0 \\
0 & 2
\end{array}\right)\left(\begin{array}{ll}
1 & 1 \\
1 & 1
\end{array}\right)\left(\begin{array}{ll}
1 & 0 \\
0 & 2
\end{array}\right)=\left(\begin{array}{ll}
1 & 2 \\
2 & 4
\end{array}\right)
$$

is line-sum-symmetric and therefore, by Corollary 5 ,

$$
u^{\mathrm{T}} A v \leqslant x^{\mathrm{T}} A y
$$

for all $(x, y) \in \mathbb{R}^{n} \times \mathbb{R}^{n}$ where $x, y \gg 0$ and $x_{i} y_{i}=u_{i} v_{i}$ for $i=1, \ldots, n$. But $u$ and $v$ are not eigenvectors of $A$.

Corollary 7 (Dantzig, Eaves and Rothblum (1985)). Let $A$ be an $n \times n$ nonnegative matrix and let $u$ and $v$ be two positive vectors in $\mathbb{R}^{n}$ where $D(u) A D(v)$ is line-sumsymmetric. Then

$$
u^{\mathrm{T}} A v \leqslant v^{\mathrm{T}} A u \text {. }
$$

Proof. As $u_{i} v_{i}=v_{i} u_{i}$ for $i=1, \ldots, n,(5.6)$ follows immediately from Corollary 5 .

\section{Patterns of matrices having line-sum-symmetric scalings}

Recall that complete reducibility is a pattern property. Hence, Theorems 1,2 and 4 (part (a) in each) imply that the property of having a corresponding line-sumsymmetric scaling is a pattern property. We next characterize such patterns.

Theorem 7, Let $A$ be an $n \times n$ nonnegative matrix whose pattern is $P$. Then the following are equivalent:

(a) $P$ is completely reducible,

(b) there exists a line-sum-symmetric matrix whose pattern is $P$,

(c) A has a line-sum-symmetric row-scaling,

(d) A has a line-sum-symmetric similarity-scaling, and

(e) A has a line-sum-symmetric equivalence-scaling.

Proof. Evidently, $P$ is completely reducible if and only if so is $A$. Hence, Theorems 1,2 and 4 (part (a) in each) imply that (a) is equivalent to (c), (d) and (e) respectively. We next show the equivalence of (b) and (a). Assume that $P$ is completely reducible. 
Then, Theorem 1 implies that $P$ has a row-scaling, say $B$, which is line-sum-symmetric and, of course, the pattern of $B$ is $P$. Next assume that $B$ is a line-sum-symmetric matrix whose pattern is $P$. As $I B=B$ is a row-scaling of $B$, Theorem 1 implies that $B$ is completely reducible, and therefore so is $P$.

The equivalence of parts (a) and (b) of Theorem 7 appears in Saunders and Schneider (1979, Theorem 2.5). Additional characterizations of patterns admitting nonnegative line-sum-symmetric matrices are given there.

\section{Decompositions of completely reducible matrices}

We next show that a square nonnegative completely reducible matrix can be decomposed into the sum of matrices which can be uniformly scaled into scalar multiples of circuit matrices.

Theorem 8. Let $A$ be an $n \times n$ nonnegative, completely reducible matrix. Then:

(a) there exist $n \times n$ nonnegative matrices $A^{1}, \ldots, A^{q}$ whose patterns are circuit matrices such that $A=\sum_{j=1}^{q} A^{j}$ and for some matrix $X$ in $\mathscr{D}_{n}, X A^{j}$ is a scalar multiple of a circuit matrix for all $j=1, \ldots, q$;

(b) there exist $n \times n$ nonnegative matrices $A^{1}, \ldots, A^{q}$ whose patterns are circuit matrices such that $A=\sum_{j=1}^{q} A^{j}$ and for some matrix $X$ in $\mathscr{D}_{n}, X A^{j} X^{-1}$ is a scalar multiple of a circuit matrix for all $j=1, \ldots, q$; and

(c) for every matrix $Y$ in $\mathscr{D}_{n}$ there exist $n \times n$ nonnegative matrices $A^{1}, \ldots, A^{q}$ whose patterns are circuit matrices such that $A=\sum_{j=1}^{q} A^{j}$ and for some matrix $X$ in $\mathscr{D}_{n}, X A^{j} Y$ is a scalar multiple of a circuit matrix for $j=1, \ldots, q$.

Proof. We establish only part (a) as the remaining parts follow from similar arguments. We first observe that Theorem 1 implies the existence of $n \times n$ diagonal matrix $X$ in $\mathscr{D}_{n}$ for which $X A$ is line-sum-symmetric. By Dantzig, Eaves and Rothblum (1985, Theorem 1), every line-sum-symmetric matrix is a linear combination with positive coefficients of circuit matrices (see also Saunders-Schneider (1979, p. 532)). Thus, there exist circuit matrices $E^{1}, \ldots, E^{q}$ and positive integers $\alpha_{1}, \ldots, \alpha_{q}$ such that $X A=\sum_{j=1}^{q} \alpha_{j} E^{j}$. It follows that $A=\sum_{j=1}^{q} A^{j}$, where for $j=1, \ldots, q, A^{j} \equiv$ $\alpha_{j} X^{-1} E^{j}$. In particular, $X A^{j}=\alpha_{j} E^{j}$.

\section{References}

A. Berman and R.J. Plemmons, Nonnegative matrices in the mathematical sciences (Academic Press, New York, 1979).

R.A. Brualdi, S.V. Parter and H. Schneider, "The diagonal equivalence of a nonnegative matrix to a stochastic matrix", Journal of Mathematical Analysis and Applications 16 (1979) 31-50. 
G.B. Dantzig, B.C. Eaves and U.G. Rothblum, "A decomposition and scaling-inequality for line-sumsymmetric nonnegative matrices", SIAM Journal of Algebraic and Discrete Methods 6 (1985) 237-241.

B.C. Eaves, "Finite solution of pure trade markets with Cobb-Douglas utilities", unpublished manuscript (1984).

G.M. Engel and H. Schneider, "Diagonal similarity and equivalence for matrices over groups with 0", Czechoslovak Mathematical Journal 25 (1975) 389-403.

G.M. Engel and H. Schneider, "Cyclic and diagonal products on a matrix", Linear Algebra and Its Applications 7 (1973) 301-335.

M. Fiedler, C.R. Johnson, T.L. Markham and M. Neumann, "A trace inequality for $M$-matrices and the symmmetrizability of a real matrix by a positive diagonal matrix", Linear Algebra and its Applications (1985), to appear.

M.v. Golitschek, U.G. Rothblum and H. Schneider, "A conforming decomposition theorem, a piecewise linear theorem of the alternative, and scalings of matrices satisfying lower and upper bounds", Mathemarical Programming 27 (1983) 291-306.

D.J. Hartfiel, "Concerning diagonal similarity of irreducible matrices", Proceedings of the American Mathematical Society 30 (1971) 413-425.

B.B. King, "What is SAM? A layman's guide to social accounting matrices", World Bank Working Paper No. 463 (1981)

A.W. Marshall and I. Olkin, "Scaling of matrices to achieve specified row and column sums", Numerische Mathematik 12 (1968) 83-90.

M.V. Menon, "Reduction of a matrix with positive elements to a doubly stochastic matrix", Proceedings of the American Mashematical Society 18 (1967) 244-247.

G. Pyatt and E. Thorbecke, Planning techniques for a better furure (I.L.O., Geneva, 1976).

B.D. Saunders and H. Schneider, "Flows on graphs and applied to diagonal similarity and diagonal equivalence for matrices", Discrete Mathematics 24 (1978) 205-220.

B.D. Saunders and H. Schneider, "Applications of the Gordan-Stiemke Theorem in combinatorial matrix theory", SIAM Review 21 (1979) 528-541.

R. Sinkhorn, "On best doubly stochastic estimates", Notices of American Society 294 (1962) 592-634.

R. Sinkhorn, "A relationship between arbitrary positive matrices and stochastic matrices" Annals of Mathematical Statistics 35 (1964) 876-879.

R. Sinkhorn and P. Knopp, "Concerning nonnegative matrices and doubly stochastic matrices", Pacific Joumal of Mathematics 21 (1967) 343-348. 